\title{
Control Pedagógico del desentrenamiento deportivo
}

\author{
Pedagogical control of sports training
}

Borys Bismark León-Reyes

Universidad Técnica de Machala bleon@utmachala.edu.ec https://orcid.org/0000-0001-6936-9947

Daniel David Hechavarria-Cardero Universidad de Oriente-Cuba dhechavarria@uo.edu.cu https://orcid.org/0000-0002-9541-7567

Geovanni Jesús Heredia-Arias gheredia@utmachala.edu.ec https://orcid.org/0000-0002-5076-8726

Melchor César León Ministerio de Educación- Dirección Distrital 07D02, Ecuador melchorleon60@gmail.com https://orcid.org/0000-0002-7466-2790

\section{Revista Cumbres Vol.7 №2}

Versión electrónica ISSN 1390-3365 http://investigacion.utmachala.edu.ec/revistas/index.php/Cumbres http://doi.org/10.48190/cumbres.v7n2a4 


\title{
RESUMEN
}

La presente investigación tiene como objetivo determinar el estado actual que presenta el control pedagógico del desentrenamiento deportivo. Se trabajó con una muestra de 29 exdeportistas, 17 entrenadores y 13 directivos seleccionados de forma aleatoria simple, pertenecientes a varias provincias de la República de Ecuador. Se realizaron entrevistas con los directivos del deporte, socialización con la comunidad científica mediante la técnica de participación PNRI y la técnica TKJ para luego delimitar los factores internos y externos según la Matriz FODA. La aplicación de los instrumentos y el análisis realizado sobre la información y datos recopilados evidencian un escaso conocimiento de los procederes teórico-metodológicos para la etapa posdeportiva y de la sistematización de las ideas surgidas en torno al control pedagógico del desentrenamiento, los cuales entorpecen la realización de acciones efectivas de desentrenamiento deportivo que asegure una reincorporación armónica de los exdeportistas a la sociedad.

Palabras clave: Atleta, Entretenimiento, Deporte, Competencia deportiva, Calidad de vida.

\begin{abstract}
The present research aims to determine the current state of the pedagogical control of sports detraining. Empirical methods and techniques were used. We worked with a sample of 29 ex-athletes, 17 coaches and 13 managers selected in a simple random way, the sample is made up of ex-athletes and coaches who belong to several provinces of the Republic of Ecuador, exchanges, meetings and interviews were held with the directors of the sport, socialize with the scientific community through the PNRI participation technique and the TKJ technique to later delimit the internal and external factors according to the SWOT Matrix, the application of the instruments and the analysis carried out on the information and data collected evidences a scarce knowledge of the theoretical-methodological procedures for the post-sport stage and the systematization of the ideas that arose around the pedagogical control of detraining, which hinder the realization of effective sports de-training actions that ensure a harmonious reincorporation of ex-athletes to society.
\end{abstract}

Keywords: Athlete, Entertainment, Sport, Sports competition, Quality of life.

\section{Cumbres}




\section{INTRODUCCIÓN}

El desentrenamiento deportivo puede ser considerado en una primera aproximación como el proceso que debe ocurrir en la etapa posdeportiva iniciada esta con el retiro del deportista donde se somete a un sistema de influencias para eliminar de forma intencionada, las adaptaciones y aprendizaje altamente especializados adquiridos durante el entrenamiento deportivo de alto rendimiento. Esto concuerda con lo planteado por Alonso (2000): “...es un proceso pedagógico, con un objetivo totalmente profiláctico para la salud del atleta que termina su vida activa en el deporte de alto rendimiento, pero que de ninguna forma significa el abandono definitivo del (...) ejercicio físico" (p.1).

Por otra parte, Aguilar (2009), tipificó al desentrenamiento como un proceso pedagógico con bases científicas, “...dirigido a la reducción sistemática, multidisciplinar e integral del nivel alcanzado por el deportista, desde una perspectiva médico-biológica, psicológica y social durante su adaptación a las nuevas condiciones del retiro con una adecuada calidad de vida" (p. 38). En esencia el desentrenamiento es un proceso donde el exdeportista debe tener una atención pedagógica sistematizada según el nivel de las potencialidades que va alcanzando (Fuentes, 2009; Frómeta, 2015).

La relación antagonista del desentrenamiento con el entrenamiento queda evidenciada en los criterios de Zamora y Frómeta (2012) cuando devela la intención del primero con la finalidad de desinstalar los beneficios o capacidades a nivel de las estructuras, funciones, sustratos y los sistemas, conseguidas en el segundo. No obstante, evidencian una dependencia profunda pero sutil.

El incremento del potencial de entrenamiento es posible gracias a periodos de desentrenamiento parcial y la cultura del desentrenamiento se afianza en el entrenamiento. Pero para esto último, se debe trabajar de forma intencionada. Al niño desde su incorporación a la práctica sistemática del deporte, se le debe inculcar la cultura por el desentrenamiento, y así en cada una de las etapas deportivas donde seguirá estando presente, porque “...se preserva solo en su desarrollo...” (Fuente et al., 2009, p.3). Si esta se mantiene estática y no progresa con el tiempo, se reduce la posibilidad de ser cultivada (Frómeta, 2015).

En los últimos años se ha incrementado la percepción del riesgo sobre los efectos del entrenamiento deportivo de alto rendimiento durante la etapa posdeportiva. Se ha anunciado con frecuencia la necesidad del desentrenamiento deportivo y demostrado en pocas ocasiones su incidencia en los exdeportistas. Los estudios (Velázquez, 1999; Alonso, 2001; Carabeo et al., 2002; Casariego, 2005; Aguilar, 2009) demostraron alteraciones fisiológicas negativas, factores de riesgos coronarios y problemas con la tensión arterial en los exdeportistas; y coincidieron en identificar como la causa más visible y principal de esas realidades, la interrupción brusca del entrenamiento de alto rendimiento. 
Pese a ello, las referencias a posibles propuestas concretas sobre el desentrenamiento no han sido abundantes. Entre las existentes (Alonso \& Forteza, 2001; Barrizontes et al., 2003; Sutherland, 2008; Carvajal, 2009; Aguilar, 2009; Barroso, 2010; Morales, 2012; Mayo, 2012; Pérez, 2012; Mustelier, 2014; Frómeta, 2015; Taupier, 2018; Mena, 2020) se encuentran las orientadas a la consideración de: concepciones teóricas, directrices básicas, modelos y principios; o la aplicación de: acciones educativas, alternativas físicas, estrategias, programas físicos, metodologías, programas. No obstante, los aportes aún carecen de la debida integralidad y con limitaciones desde su propia concepción.

Desde el orden teórico, una de las carencias fundamentales radica en la arista pedagógica pues, al igual que el entrenamiento deportivo en sus inicios, se centra en los aspectos biológicos, en este caso de naturaleza: biomédicos y psicopatológicos y no en lo formativo ni lo cultural. Un ejemplo de ello es la concepción de Mujica (2010), quien reconoció al desentrenamiento como: “...la pérdida parcial o completa de las adaptaciones anatómicas, fisiológicas y de rendimiento, producida por el entrenamiento, como consecuencia de una reducción o cese del entrenamiento" (p.1). Aquí se puede apreciar con claridad, la desestimación de aspectos formativos.

Aunque sin la certeza de haberse realizado un análisis sistémico para entender la naturaleza del desentrenamiento, varios autores (Alonso, 2000; Sutherland, 2008; Carvajal, 2009; González \& Torregosa, 2009; Santana, 2010; Crusellas, 2012) los clasifican como un proceso pedagógico, pero sin una clara fundamentación.

En este sentido pocos investigadores ahondan en la naturaleza pedagógica del desentrenamiento, tal como lo hace Frómeta (2015). Según la propia investigadora, este proceso, presenta un cuerpo teórico sostenido en los fundamentos de una pedagogía especial, convertida a su vez en contenido para la educación como fenómeno social. La concepción del desentrenamiento desde esta perspectiva, implica considerar dos elementos básicos: la formación de una cultura y la proyección praxeológica.

No obstante, quedan aún otras insuficiencias fundamentales como es el caso del control de este proceso pues los sistemas de referencias para realizar las comparaciones durante la retroalimentación aún no presentan el nivel de sistematicidad requerida para que sean útiles y la naturaleza pedagógica no se evidencia con claridad pues solo se enfocan en los estándares a cumplir y no en el aprovechamiento de las potencialidades de los exdeportistas.

El control solo es visto con la finalidad de evaluar el estado de salud, físico y mental, los acontecimientos ocurridos durante la vida activa del deportista (lesiones, traumas y otros), con el fin de desarrollar y ajustar las tareas del programa de desentrenamiento físico reales a las características de cada exdeportista. Para ello se apoyan en indicadores propuestos en investigaciones antecedentes (Barroso, 2010; Mayo, 2012). Esta limitación no aprecia con mayor claridad si se asume, tal como lo hacen los autores de este trabajo, al desentrenamiento como el proceso de reorientación de adaptaciones y apren- 
dizaje muy especializado a otros más generales y diversificado con mayor utilidad en las tareas habituales del ser humano; y de la trasformación de una cultura del rendimiento por otra formativa de extensión e intensificación de la calidad de vida.

Ambas limitaciones se traducen en la necesidad imperativa de un primer acercamiento de ambos elementos y para lo cual se propone determinar el estado actual que presenta el control pedagógico del desentrenamiento deportivo.

\section{MATERIALES Y MÉTODOS}

Se seleccionó mediante el método básico aleatorio simple una muestra probabilística de 29 exdeportistas, 17 entrenadores y 13 directivos pertenecientes a varias provincias de la República de Ecuador. Como criterio de inclusión de la población se determinó: tener una trayectoria en el proceso de entrenamiento deportivo de alto rendimiento y oficializar su retiro deportivo definitivo parte de los exdeportistas. en el caso de los criterios de exclusión se desestimó a aquellos deportistas con menos de 5 años en el alto rendimiento y los que presentan patologías que les impide la realización de la actividad física. La unidad de análisis se enmarca en el personal con responsabilidad directa al desentrenamiento deportivo como proceso sucediente al entrenamiento de alto rendimiento.

El estudio se desarrolló desde julio a finales de septiembre del 2020. Los métodos, técnicas e instrumentos se ajustan a los requerimientos propuestos por Frómeta (2015). Para ello se realizó un diagnóstico a través de intercambio, reuniones y entrevistas con los directivos del deporte, socializar con la comunidad científica mediante la técnica de participación PNRI y la técnica TKJ para luego delimitar los factores internos y externos según la Matriz FODA. Se aplicaron en la modalidad virtual en su mayoría y solo en algunos casos de forma presencial.

Las variables estudiadas fueron dinámica del control pedagógico actual del desentrenamiento deportivo y el conocimiento existente sobre los componentes personológicos del desentrenamiento y el personal gestor del mismo.

\section{RESULTADOS}

Con el objetivo de conocer la orientación recibida sobre el desentrenamiento parcial y total, el estado de salud física y mental, el conocimiento y el estilo de vida mantenido durante el retiro. El 75,86\% no realizó el desentrenamiento y no recibió orientación al respecto; el 24,13\% expresó haber efectuado actividades dirigidas por los entrenadores, sin embargo, en su totalidad no pudieron profundizar en las características de ese proceso. 
El 62,06\% del total manifestó padecer de algún problema de salud. Destacaron los cardiovasculares y de estos las cardiopatías. El 20,68\% sufre de enfermedades crónicas no transmisibles, con prevalencia de la hipertensión arterial. El 17,24\% sufre de trastornos osteo-articulares. Con mucha menor incidencia se reportaron las enfermedades del sistema nervioso central y afecciones de la piel.

En la totalidad de la muestra se apreció tener una vida no estructurada, el 55,17\% refirió estar bajo tratamiento medicamentoso o terapéutico y el $44,82 \%$ se automedica cuando sufre alguna crisis. El 34,48\% se consideró como practicantes asiduos de actividades físicas y el 58,62\% se declaró ser sedentario, aunque desea incorporarse a su práctica regular y dirigida.

Al 68,95\% de los entrenadores de alto rendimiento, se les aplicó una encuesta para diagnosticar sus conocimientos sobre el control pedagógico del desentrenamiento. El 79,31\% conoce el proceso investigado, sin embargo, existe una vaga noción al exponer los argumentos solicitados y el 20,68\% manifiesta no conocerlo.

El 82,75\% expresó no estar preparado para realizar un adecuado control pedagógico del desentrenamiento. El 17,24\% se creyó incapacitado para hacerlo. El 51,72\% opinó que ellos pueden ser los responsables del control pedagógico del desentrenamiento de los exdeportistas. El 100\% declaró no haber recibido nunca preparación, ni superación sobre el control pedagógico del desentrenamiento, pero mostró interés por ser instruidos en esa dirección.

En resumen, se puede apreciar la existencia de un exiguo conocimiento para la dirección y la orientación del desentrenamiento. Esto hasta cierto punto, permite evadir la responsabilidad con ese proceso, al no encontrarse entre ellos los máximos responsables en el problema investigado.

La encuesta a directivos reveló que el 100\% convino en plantear que no se desarrollan actividades concernientes al control pedagógico del desentrenamiento deportivo. El 79,31\% desconoció la existencia de documentos donde se indique su realización como proceso. El 86,2\% apreció que no desarrollan actividades en función del control pedagógico del desentrenamiento, debido a la no existencia de un personal responsabilizado con esa actividad.

El análisis de la matriz FODA identificó como debilidades: exigua presencia del tema sobre el control pedagógico del desentrenamiento en la superación de los entrenadores deportivos; existencia de documentos normativos que no cumplen las exigencias teórico-metodológicas para desarrollar el control pedagógico del desentrenamiento; falta de una sólida política para la solución al problema, inexistencia del personal responsabilizado con el control pedagógico del desentrenamiento; y poco conocimiento de deportistas, entrenadores, directivos, médicos, psicólogos, familiares y otros profesionales, sobre el tema investigado.

Entre las fortalezas se encuentran: conciencia política para crear los elementos de gestión y solucionar la problemática existente; existencia de entrenadores con un alto conocimiento de sus profesiones; y reconocimiento por el personal a cargo de la preparación del deportista, de lo útil de ofrecer vías para solucionar ese problema agudizado por años. 
Entre las oportunidades se encuentra la intencionalidad institucional para favorecer la implementación de las propuestas diseñadas para resolver esta limitación en el proceso de desentrenamiento deportivo. Como amenazas: dificultades con la obtención de recursos humanos, financieros y materiales, para garantizar la calidad del desentrenamiento, con todos los requerimientos necesarios como los dedicados al proceso de entrenamiento; e insuficiente cultura adquirida por los profesionales comprometidos con la preparación del deportista, para el control del desentrenamiento.

Teniendo en cuenta las insuficiencias detectadas, se aplicó la técnica PNRI. De los criterios sugeridos, tanto positivos como interesantes, superaron a los negativos, los cuales en ninguno de los casos se refirieron a considerar absurdo el hecho de asumir la idea del control pedagógico como parte del desentrenamiento. Las opiniones giraron en torno a las dificultades para aceptarlo como tal y los obstáculos para concretarlo, pues tenían el convencimiento de la importancia de este.

Si se atiende a las exigencias de la técnica empleada, se pudiera creer en opiniones opuestas al desentrenamiento, sin embargo, no ocurrió así, pues todos los entrenadores se convencieron de la utilidad de ponerlo en práctica por el impacto en la calidad del estado de salud de los exdeportistas en el retiro.

Entre los criterios positivos sobresale por la complejidad y especificidad del control pedagógico del desentrenamiento deportivo, la demanda en la creación de su propia teoría y metodología, para asegurar los principios, métodos, procedimientos de control y evaluación, mediante el desarrollo de estudios científicos sistematizado.

Existe total coincidencia en garantizar la reinserción de los exdeportistas a la vida cotidiana, al constituirse una demanda social, por ello, el control de este proceso adquiere vital importancia.

El análisis e interpretación de los criterios negativos, apuntaban a ir en contra del proceso investigado, y sí a los obstáculos para su materialización, por eso, los entrenadores expresaron, no conocer la proyección nacional sobre el impacto negativo en la población de exdeportistas por no haber tenido, en unos casos, el futuro garantizado con el desentrenamiento.

Se lamentaron por no haber recibido, ninguna instrucción sobre la teoría del desentrenamiento. Incluso ven como un freno la exigua proyección en el pregrado y el posgrado, pues no se asumen con la fuerza necesaria, como algo capital, el acceso a la información es insuficiente y en eso deben trabajar la institución deportiva y su universidad.

Los criterios interesantes constituyen estímulos positivos para cualquier proyecto. Los entrenadores al emitir sus juicios sobre el control pedagógico del desentrenamiento, reconocieron la novedad y actualidad del tema, por eso, la mayoría de ellos vio con agrado haber decidido empezar su socialización entre los trabajadores del deporte, pues son saberes poco frecuentes en el ámbito deportivo. Reconocieron la novedad de la experiencia, pues hasta ahora no había sido una cuestión de interés para el debate profesional, a pesar de su sensible importancia. 
Hubo conformidad en constituirse en una preocupación para los directivos teniendo en cuenta su necesidad práctica. Para los entrenadores resultó muy interesante tratar de aunar todos los criterios científicos posible, para estudiar de manera sistemática ese fenómeno.

Las recomendaciones formuladas en sentido general son importantes, pero a los efectos de su mayor comprensión. Los entrenadores se pronunciaron de forma general por crear grupos de entrenadores para dirigir el proceso, sistematizando todos los saberes acerca del tema. Esta idea es vital, porque el problema no es abrazar al desentrenamiento como algo novedoso. Se debe tener en cuenta la diversidad de disciplinas deportivas y las particularidades individuales de los practicantes, por consiguiente, esa especificidad también debe ser expresada en la capacitación de entrenadores.

La otra recomendación muy atinada consistió en establecer indicadores sobre el estado actual de los deportistas en lo físico, médico, psicológico, intelectual y sociológico y otros, cuando pasan al retiro para iniciar el desentrenamiento.

Se desarrolló un taller de construcción colectiva a través de la técnica de participación TKJ con los 13 directivos con el objetivo de determinar las necesidades de aprendizaje sobre el control pedagógico del desentrenamiento.

A partir de la obtención de cada uno de los resultados dados por los participantes a los facilitadores (quien aplicó la técnica TKJ), se concretan las temáticas a tratar sobre el control pedagógico. Este procedimiento permitió definir las necesidades educativas colectivas e individuales con mayores prioridades. Las cuales versan sobre los indicadores de control, la conformación de estudios longitudinales y de series cronológicas para precisar los comportamientos que fungirán como sistema de referencias durante el control, la visualización del estado inicial y la indagación de las potencialidades de los exdeportistas y la valoración del aprendizaje sobre nuevos estilos de vida.

\section{DISCUSIÓN}

Los resultados obtenidos confirman las alteraciones fisiológicas con riesgos coronarios y problemas con la tensión arterial en los exdeportistas (Velázquez, 1999; Alonso, 2001; Carabeo et al., 2002; Casariego, 2005; Aguilar, 2009). Por otro lado, fue evidente la carente integralidad en la concepción y desarrollo de este proceso concordando así con los resultados de Frómeta (2015) siendo la más afectada, la dimensión pedagógica.

En cuanto al conocimiento y concientización de la necesidad del proceso de desentrenamiento, se manifiesta un estado similar a lo localizado en otras regiones tal como es el caso de Cuba, país destacado en la esfera deportiva. esta concordancia induce a considerar que existe una tendencia creciente a ponderar la necesidad de implementar este proceso sobre bases teóricas sólidas desde una concepción holística que garanticen las herramientas científicas de actuación en la práctica y el incremento de la calidad formativa de los profesionales que atenderán este proceso.

\section{Cumbres}


El diagnóstico reveló las insuficiencias en cuanto al control pedagógico del desentrenamiento, la inexistencia de una estructura metodológica capaz de garantizar la continuidad de las actividades físicas, manifestada en la limitada orientación de los exdeportistas a la realización del desentrenamiento, dificultándose su reincorporación a la sociedad con estilos de vida saludables.

\section{REFERENCIAS BIBLIOGRÁFICAS}

Aguilar, E. M. (2009). Metaprograma de entrenamiento físico para el desentrenamiento deportivo de atletas de élite. [Tesis de doctorado no publicada]. La Habana. Iscf "Manuel Fajardo" (Soporte digital)

Alonso, R. F. (2000). La medicina deportiva en el entrenamiento deportivo (III). Desentrenamiento deportivo: teoría o hipótesis. EFDeportes.com. [Revista Digital]. Año 5. $\mathrm{N}^{\circ}$ 25. Disponible en: http://www.efdeportes. com/. Consultado: mayo 2013

Alonso, R. F. (2001). Desentrenamiento deportivo: análisis y criterios actuales. EFDeportes.com. [Revista Digital]. Año 7. N³7. Junio. Disponible en: http://www.efdeportes.com/. Consultado: mayo 2014

Alonso, R. F. y Forteza, A. y López, R. (2001). Principios metodológicos del desentrenamiento deportivo. EFDeportes.com. [Revista Digital]. Año 7. № 40. Disponible en: http://www.efdeportes.com/. Consultado en: mayo 2013

Barrizonte, F. y col. (2003). Desentrenamiento deportivo en Cuba. Instituto de Medicina del Deporte de Cuba. La Habana (Soporte digital)

Barroso, P. M. (2010). Indicadores para la caracterización del retiro deportivo el atletismo de alto rendimiento: un criterio para la individualización del desentrenamiento deportivo. [Tesis doctoral no publicada]. La Habana. Uccfd "Manuel Fajardo"

Carabeo, A. I. y col. (2002). Propuesta de actividades para confeccionar un programa de desentrenamiento deportivo para ciclistas de la modalidad ruta. EFDeportes.com. [Revista Digital]. Año 8. $\mathrm{N}^{\circ}$ 46. Disponible en: http://www.efdeportes.com/. Consultado en: mayo 2013

Carvajal, M. (2009). Desentrenamiento deportivo parcial, una alternativa para entrenadores del área de velocidad. [Tesis de maestría no publicada]. Santiago de Cuba. Uccfd. "Manuel Fajardo"

Casariego, C. (2005). Indicadores psicológicos. Su importancia en la etapa de culminación del deporte activo. La Habana: Cepromede

Crusellas, A. (2012). Desentrenamiento deportivo de los atletas retirados. [Tesis de especialidad para el deporte de alto rendimiento no publicada]. Pinar del Río. Uccfd "Manuel Fajardo"

Frómeta, N. (2015). Estrategia pedagógica para la proyección del desentrenamiento deportivo. Tesis doctoral. Holguin: Universidad de Ciencias de la Cultura Física y el Deporte 
Fuentes, H. C. (2009). Concepción científica holístico-configuracional. Santiago de Cuba: CeeS "M.F. Gran” - Universidad de Oriente.

González, M. D. y Torregrosa, M. (2009). Análisis de la retirada de la competición de élite: antecedentes, transición y consecuencias. Revista Iberoamericana de Psicología del Ejercicio y el Deporte. 4(1), 93-104

Mayo, M. y col. (2012c). Un acercamiento a los fundamentos teóricos para la individualización del desentrenamiento deportivo. Primera parte. EFDeportes.com, [Revista Digital]. Buenos Aires. Año 17. № 168. Disponible en: http://www.efdeportes.com. Consultado en: agosto 2014

Mena Pérez, O. (2020). Modelo para la planificación del proceso de desentrenamiento deportivo. Revista Conrado, Vol 16, Num 75, 8-14

Morales, A. (2012). Principios para la individualización del desentrenamiento deportivo: profundización y acercamiento a sus fundamentos. Memorias de Cidar-2012. Santiago de Cuba. Uccfd "Manuel Fajardo" (Soporte digital)

Mustelier, A. (2014). Acciones educativas encaminada al período de tránsito de los jokistas juveniles. [Trabajo de diploma no publicado]. Santiago de Cuba. Uccfd. "Manuel Fajardo"

Pérez, Y. (2012). La individualización del entrenamiento y el desentrenamiento deportivo. Multimedia para la superación profesional. [Trabajo de diploma no publicado]. Villa Clara. Uccfd "Manuel Fajardo"

Santana, J. L. (2010). El Desentrenamiento deportivo en la vida del deporte de alto rendimiento. EFDeportes.com. [Revista digital]. Disponible en: http:// www.efdeportes.com.Año13, №129.Consultado en: enero2012

Sutherland, E. J. (2008). Planificación de un conjunto de actividades para el desentrenamiento deportivo en maratonistas retirados. [Tesis de maestría no publicada]. Santiago de Cuba. Uccfd "Manuel Fajardo"

Taupier, G. (2018). Metodología para el desentrenamiento en exatletas juveniles del deporte levantamiento de pesas. EmásF, Revista Digital de Educación Física. Año 9, Num. 50, p. 10-18

Velázquez, J. (1999). Desentrenamiento deportivo: alternativa vital. Revista Bohemia. La Habana. Año 91. № 5

Zamora, R. y Frómeta, N. (2012). El deportista de alto rendimiento: apuntes para pensar en su desentrenamiento deportivo. Memorias. II Convención Internacional del Deporte de Alto Rendimiento. Santiago de Cuba "Manuel Fajardo" 